\section{Vitreoretinal surgery after childhood ocular trauma}

RM Sheard, K Mireskandari, E Ezra and

PM Sullivan

Keywords: child; adolescent; eye injuries; retinal detachment; vitrectomy; scleral buckling

\section{Introduction}

Children aged under 10 years account for $6.5 \%$ of all eye injuries, ${ }^{1}$ but $21-35 \%$ of hospital admissions for eye trauma are from the paediatric age group. ${ }^{2-4}$ Children are overrepresented among individuals with serious eye injuries, accounting for approximately one-third of cases. ${ }^{5,6}$ Most surgeons agree that vitreoretinal surgical techniques allow many poor prognosis eyes to be salvaged, ${ }^{7,8}$ but trauma remains a leading cause of monocular blindness in children. ${ }^{9}$ We wish to report the indications for, complications of, and outcomes of vitreoretinal intervention in the management of childhood ocular trauma.

\section{Methods}

We reviewed the case notes of all children aged 16 years or younger undergoing vitreoretinal surgery (either pars plana vitrectomy or scleral buckling) at Moorfields Eye Hospital between March 1991 and September 1999. From these, we identified those children with a history of ocular trauma for detailed review. Data collected included demographics, presenting examination findings, prior ocular surgery, operative details and complications, and outcome.

We classified the type of injury according to the standardised classification of ocular trauma proposed by Kuhn et $a l^{10}$ (Figure 1). We analysed the data to determine the indications for vitreoretinal surgical intervention, its complications, and outcomes. We also performed univariate analysis to identify factors associated with a poor visual outcome.

Snellen visual acuities (VA) were recorded using the following scale: $6 / 5,6 / 6,6 / 9,6 / 12$, $6 / 18,6 / 24,6 / 36,6 / 60$, counting fingers $(C F)$, hand movements, perception of light, and no perception of light (NPL). We defined the VA of enucleated eyes as NPL. Data were compared
Moorfields Eye Hospital, City Road, London, UK

Correspondence: RM Sheard, Department of Ophthalmology, A Floor, Royal Hallamshire Hospital, Glossop Road, Sheffield S10 2JF, UK Tel: + 44114271 3829; Fax: + 441142713682

E-mail: richard@

richardsheard.net

Received: 12 July 2005 Accepted in revised form: 10 January 2006 Published online: 7 April 2006

Conflicts of interest: None Proprietary interests: None

Funding: None 


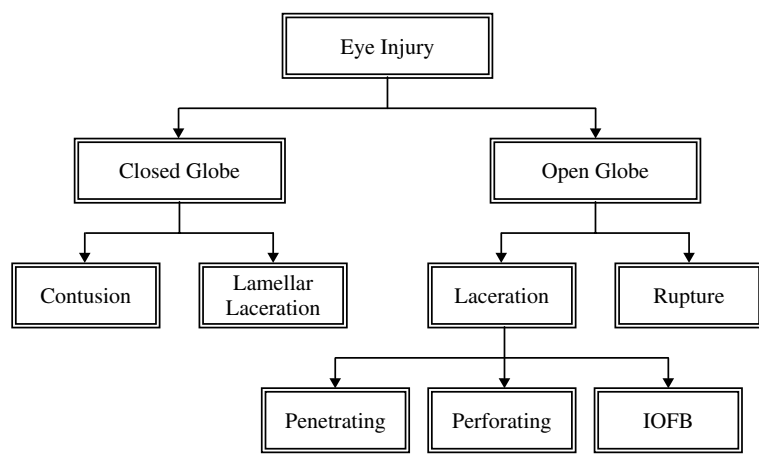

Figure 1 Classification of ocular trauma (after Kuhn $e a^{11}$ ).

using non-parametric statistical methods. If a calculated VA lies between two points on the scale, we quote the lower point followed by the plus symbol, for example 6/ $60+$.

The chi-squared test $\left(\chi^{2}\right)$ was used to compare proportions between groups, or the Fisher exact test when any cell in the contingency table was less than five. The two-tailed unpaired $t$-test was employed for comparing continuous variables between groups, the Mann-Whitney U-test for comparing discrete variables between groups, and the Wilcoxon rank sum test for comparing paired discrete variables. A $P$-value of less than 0.05 was considered significant.

\section{Results}

We identified 61 children who underwent vitreoretinal surgery following ocular trauma. There were 49 boys and 12 girls (4.1:1 ratio) with a mean age of 11.1 years (range 18 months to 16 years). All the patients had the initial management of their eye injury by ophthalmologists outside the Moorfields Vitreoretinal Service and were subsequently referred for further assessment.

Twenty-eight children (45.9\%) had open globe injuries (OGI); there were two cases of intra-ocular foreign body (IOFB), two perforating injuries, 23 penetrating injuries, and one globe rupture. Thirty-three children $(54.1 \%)$ had contusive closed globe injuries (CGI). All injuries were unilateral. Table 1 summarises the demographics of the two groups.

One patient with OGI and seven patients with CGI had pre-existing ocular or systemic diagnoses (OGI: EhlersDanlos syndrome; CGI: retinopathy of prematurity two cases, congenital glaucoma, high myopia, Marfan's syndrome, Stickler's syndrome and, Cornelia de Lange syndrome - one case each).

All eyes with OGI had had primary surgical repair before referral. In three the crystalline lens was lost at the time of injury, in a further two a lensectomy was performed and one of these had a primary intra-ocular lens implant.
Table 1 Demographics of children with eye injuries

\begin{tabular}{lccc}
\hline & $\begin{array}{c}\text { Open globe } \\
\text { injuries } \\
\mathrm{n}=28\end{array}$ & $\begin{array}{c}\text { Closed globe } \\
\text { injuries } \\
\mathrm{n}=33\end{array}$ & P-values \\
\hline $\begin{array}{l}\text { Mean age (SD) } \\
\text { Boys : girls }\end{array}$ & $9.5(4.1)$ & $12.3(2.8)$ & $0.0068^{\mathrm{a}}$ \\
\% Boys & $21: 7$ & $28: 5$ & $0.3349^{\mathrm{b}}$ \\
\hline
\end{tabular}

$\mathrm{SD}$, standard deviation.

${ }^{\text {aUnpaired }} t$-test.

${ }^{\mathrm{b}} \chi^{2}$ test.

Of the eyes with CGI, three had undergone retinal re-attachment surgery at the referring hospital (two scleral buckling procedures, one vitrectomy) and two had undergone cataract extraction, one with lens implant.

\section{Preoperative findings}

Clinical findings at presentation are summarised in Table 2. Retinal detachment (RD) was present in 17 eyes after OGI $(60.7 \%)$ and in 30 following CGI $(90.9 \%$, Fisher exact test: $P=0.0067)$. There was no difference between the groups with respect to macular involvement in the detachment, the presence of proliferative vitreoretinopathy (PVR), or the presence of vitreous haemorrhage. Children with OGI were significantly more likely to have cataract or lens subluxation than those with CGI (57.1 vs 6.1\%, Fisher exact test: $P<0.0001$ ).

We identified three subgroups of children. Group A are those with traumatic RD treated with scleral buckling, group $\mathrm{B}$ are those with traumatic RD treated with vitrectomy, and group $\mathrm{C}$ are those with attached retinas who underwent vitrectomy for other trauma-related indications.

\section{Traumatic retinal detachment}

Children with traumatic RD after OGI were significantly more likely to undergo vitrectomy than those with traumatic RD after CGI. Sixteen of the 17 children with $\mathrm{RD}$ after OGI underwent pars plana vitrectomy (94.1\%), compared to 14 of the 30 with RD resulting from CGI (46.7\%, Fisher exact test: $P<0.0001)$.

\section{Group A: scleral buckling for traumatic retinal detachment}

In total, 17 children underwent scleral buckling surgery for traumatic RD (27.9\%). Indications for scleral buckling were absence of cataract, minimal vitreous haemorrhage or haze, single retinal break (four eyes, $23.5 \%$ ), retinal dialysis (12 eyes, $70.6 \%$ ), and absence of PVR. In all cases, a circumferential silicone explant was placed, and none were encircled. Subretinal fluid was drained in nine 
Table 2 Ocular complications at presentation

\begin{tabular}{lccc}
\hline & All injuries $(\mathrm{n}=61)$ & Open globe injuries $(\mathrm{n}=28)$ & Closed globe injuries $(n=33)$ \\
\hline Retinal detachment & $47(77.0 \%)$ & $17(60.7 \%)$ & $30(90.9 \%)$ \\
Macula-off detachment $^{\mathrm{a}}$ & $31(66.0 \%)$ & $10(58.8 \%)$ & $21(70.0 \%)$ \\
Traumatic cataract $^{\text {Vitreous haemorrhage }}$ & $16^{\mathrm{b}}(26.2 \%)$ & $14^{\mathrm{b}}(50.0 \%)$ & $2(6.1 \%)$ \\
Aphakic/pseudophakic & $13(21.3 \%)$ & $8(28.6 \%)$ & $5(15.1 \%)$ \\
PVR Grade C or worse & $7(11.5 \%)$ & $5(17.8 \%)$ & $2(6.1 \%)$ \\
Endophthalmitis $_{\text {Lens subluxation or dislocation }}^{\mathrm{a}}$ & $5(10.6 \%)$ & $2(11.8 \%)$ & $3(10.0 \%)$ \\
Intraocular foreign body & $4(6.6 \%)$ & $4(14.3 \%)$ & \\
Macular hole & $3^{\mathrm{b}}(4.6 \%)$ & $3^{\mathrm{b}}(10.7 \%)$ & $2(7.1 \%)$ \\
\hline
\end{tabular}

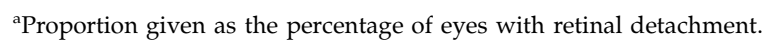

${ }^{b}$ One cataractous lens also subluxed.

eyes $(52.9 \%)$ and one eye had a D-ACE procedure (drainair-cryopexy-explant sequence). Surgery was successful after the first procedure in 12 eyes $(70.6 \%)$, after the second in another four eyes $(23.5 \%)$, and in the final case the retina was re-attached after a total of four procedures for PVR, including intra-ocular tamponade with silicone oil.

\section{Group B: vitrectomy for traumatic retinal detachment}

Thirty eyes underwent vitrectomy for traumatic RD (49.2\%). Indications for vitrectomy were prior failed vitreoretinal surgery, cataract or lens subluxation, vitreous haemorrhage or haze, multiple retinal breaks, posterior retinal breaks, giant retinal tear, PVR, retinal or vitreous incarceration, and intragel abscess. The agent used for retinal tamponade was sulphur hexafluoride gas $\left(\mathrm{SF}_{6}\right)$ in five eyes, perfluoropropane gas $\left(\mathrm{C}_{3} \mathrm{~F}_{8}\right)$ in five eyes and silicone oil in 20 eyes. There was no significant difference in the ages of children receiving oil or gas tamponade (unpaired $t$-test: $P=0.3531$ ). Primary success was achieved in 14 eyes $(46.7 \%)$, but 16 retinas re-detached $(53.3 \%), 13$ as a result of PVR. No further detachment surgery was performed in six cases, and the other 10 eyes underwent a median of two more vitreoretinal procedures (range 1-5). Ultimately, complete retinal re-attachment was achieved in 17 eyes (56.7\%, two with continued silicone oil tamponade) and re-attachment at the posterior pole in another nine eyes (30.0\%, four with silicone oil tamponade). Retinal re-attachment surgery failed in four eyes (13.3\%), and two of these eyes became phthisical.

\section{Group C: vitrectomy for other trauma-related indications}

Fourteen children had attached retinas but underwent vitrectomy for other indications related to the eye injury (22.9\%). These included cataract or lens subluxation, vitreous haemorrhage or haze, removal of intra-ocular foreign body, or macular hole. One eye developed retinal detachment with PVR postoperatively (5.9\%) and despite further surgery became phthisical.

\section{Proliferative vitreoretinopathy}

PVR was observed at presentation in five eyes, and a further 12 eyes developed new PVR after the first surgical procedure. PVR therefore affected 17 of the 61 eyes studied (27.9\%), or $35.4 \%$ of the 48 eyes that had or developed retinal detachment, and was the cause of 15 of the 22 re-detachments $(68.2 \%)$. An additional four eyes $(6.6 \%)$ developed epiretinal membranes on attached retina.

\section{Visual outcomes}

The patients were followed for a mean of 19.6 months (SD 19.0 months, median 12.5 months). Two patients were followed for less than a month because they had travelled from distant referral centres for surgery and were subsequently reviewed locally. These two patients and another three with incomplete VA data are not included in the analysis of visual outcome.

Table 3 summarises the visual outcomes of 56 of the 61 children requiring vitreoretinal surgery after eye trauma. The median VA of all the patients together improved from $C F$ at presentation to $6 / 36$ at final follow-up (Wilcoxon signed rank test: $P=0.0031$ ).

Children with OGI had a median VA at presentation of $\mathrm{CF}$, significantly worse than those with CGI (median VA 6/60, Mann-Whitney U-test: $P=0.0016$ ). Following vitreoretinal surgery, the median VA both groups improved to $6 / 36$ (Wilcoxon signed rank test: $P=0.0082$ for OGI and $P=0.1068$ for CGI). There was no statistically significant difference in the final VA of these two groups (Mann-Whitney U-test: $P=0.2044$ ). 
Table 3 Visual outcomes after vitreoretinal surgery for eye injuries

\begin{tabular}{|c|c|c|c|}
\hline & $\begin{array}{l}\text { All injuries } \\
(\mathrm{n}=56)\end{array}$ & $\begin{array}{l}\text { Open globe injuries } \\
\qquad(\mathrm{n}=25)\end{array}$ & $\begin{array}{l}\text { Closed globe injuries } \\
\qquad(\mathrm{n}=31)\end{array}$ \\
\hline \multicolumn{4}{|l|}{ Median VA } \\
\hline Preoperative & $\mathrm{CF}$ & $\mathrm{CF}$ & $6 / 60$ \\
\hline Final & $6 / 36$ & $6 / 36$ & $6 / 36$ \\
\hline Improved & $32(57.1 \%)$ & $15(60.0 \%)$ & $17(54.8 \%)$ \\
\hline Unchanged & $11(19.6 \%)$ & $6(24.0 \%)$ & $5(16.1 \%)$ \\
\hline Worse & $13(23.2 \%)$ & $4(16.0 \%)$ & $9(29.0 \%)$ \\
\hline \multicolumn{4}{|l|}{ Final VA } \\
\hline$\geq 6 / 60$ & $37(66.1 \%)$ & $15(60.0 \%)$ & $22(71.0 \%)$ \\
\hline \multirow[t]{2}{*}{$\geq 6 / 12$} & $13(23.2 \%)$ & $6(24.0 \%)$ & $7(22.6 \%)$ \\
\hline & $\begin{array}{l}\text { Group A Scleral buckle for } \\
\text { traumatic } R D(\mathrm{n}=17)\end{array}$ & $\begin{array}{l}\text { Group B Vitrectomy for } \\
\text { traumatic } R D(\mathrm{n}=28)\end{array}$ & $\begin{array}{l}\text { Group C Vitrectomy for } \\
\text { other indications }(\mathrm{n}=11)\end{array}$ \\
\hline \multicolumn{4}{|l|}{ Median VA } \\
\hline Preoperative & $6 / 36$ & $\mathrm{CF}$ & $\mathrm{CF}$ \\
\hline Final & $6 / 24$ & $\mathrm{CF}$ & $6 / 18$ \\
\hline Improved & $11(64.8 \%)$ & $10(35.7 \%)$ & $11(100.0 \%)$ \\
\hline Unchanged & $3(17.6 \%)$ & $8(28.6 \%)$ & 0 \\
\hline Worse & $3(17.6 \%)$ & $10(35.7 \%)$ & 0 \\
\hline \multicolumn{4}{|l|}{ Final VA } \\
\hline$\geq 6 / 60$ & $15(88.2 \%)$ & $12(42.9 \%)$ & $10(90.9 \%)$ \\
\hline$\geq 6 / 12$ & $6(35.3 \%)$ & $2(7.1 \%)$ & $5(45.4 \%)$ \\
\hline
\end{tabular}

\section{Prognostic factors}

We performed univariate analysis to identify factors associated with a poor visual outcome, defined as a final VA of CF or worse. There was no significant association between outcome and the following factors: patient age, type of injury, the presence of lens injury, vitreous haemorrhage or endophthalmitis, and requirement for more than one vitreoretinal surgical procedure. The association between poor outcome and presence of RD at presentation approached, but did not reach, statistical significance (Fisher exact test: $P=0.0769$ ).

Preoperative VA of CF or worse was associated with a poor outcome $\left(\chi^{2}: P=0.0306\right)$, as was the necessity for vitrectomy in the presence of traumatic RD (Fisher exact test: $P=0.0003)$. The presence or development of PVR was significantly associated with poor outcome (Fisher exact test: $P<0.0001)$. The presence of partial or total RD at final follow-up was associated with a poor visual outcome (Fisher exact test: $P=0.0001$ ), as was the retention of silicone oil in the eye (Fisher exact test: $P=0.0002$ ).

\section{Discussion}

We have described a series of 61 children selected by their need for vitreoretinal surgery after ocular trauma.
The $4: 1$ ratio of boys to girls in this study is similar to that reported in other series. ${ }^{3,9,11-13}$ The mean age of children with OGI was 9.5 years, comparable to other reports; ${ }^{11-13}$ however, we found that children with CGI were significantly older (mean age 12.3 years, $P=0.0068$ ). The majority of injuries in children under 10 years result from blows or falls, whereas the leading cause after this age is sport, ${ }^{9}$ possibly resulting in different patterns of injury, and this may explain the observed age difference.

Seven of our patients had ocular diagnoses associated with an enlarged or weakened globe that rendered the eye vulnerable to trauma (Ehlers-Danlos syndrome, retinopathy of prematurity, high myopia, Marfan's syndrome, and Stickler's syndrome). Another patient had repeated trauma as a result of 'head-banging' associated with Cornelia de Lange syndrome.

The children in this study were referred from other hospitals and so the details of the extent of the ocular injury were not available in many cases. We were therefore unable to grade the severity of the injury according to the Ocular Trauma Classification Group (OTCG) terminology ${ }^{14}$ and instead we used the simpler classification of Kuhn et al ${ }^{10}$

Children with OGI had significantly more lens injury than those with CGI (64.3 vs 6.1\%, $P<0.0001)$. This may suggest that OGI is more likely to cause damage to the 
lens or zonules, but in this selected series may also imply that lens injury after OGI is more likely to require vitreoretinal management than after CGI.

A significantly higher proportion of children in the CGI group had RD (90.9\%) than the OGI group (60.7\%, $P=0.0067)$; however, this difference may simply reflect the additional indications for vitreoretinal intervention in the OGI group, such as IOFB and lens injury.

Of the children with traumatic RD, those with OGI were more likely to require vitrectomy than those with CGI (94.1 vs $46.7 \%, P<0.0001)$. Retinal dialysis is the commonest type of retinal break seen after CGI, ${ }^{15,16}$ responsible for more than half of RDs after this mechanism of injury, and tractional horseshoe tears account for about another $20 \% .{ }^{17}$ Scleral buckling techniques are therefore likely to be successful in a high proportion of cases of RD after CGI. Retinal breaks after OGI may be large or posteriorly located, or result from fibrocellular proliferation and contraction within the vitreous. ${ }^{18}$ Vitrectomy techniques are therefore more likely to be required in these cases.

Group A consisted of 17 children who underwent a scleral buckling procedure. We used segmental circumferential solid silicone explants in all cases and no eye was encircled. Subretinal fluid was drained in a high proportion of cases $(52.9 \%)$, similar to the rate reported by Häring $e t$ al $^{19}$ Retinal re-attachment was achieved after one operation in $70.6 \%$ of cases, and this primary success rate is lower than the $88 \%$ reported in other series. ${ }^{19,20}$ However, only $24-56 \%$ of detachments in these reports were following trauma, and different surgical techniques were used, including placement of an encircling band. There was a statistically significant improvement in median VA from $6 / 36$ to $6 / 24(P=0.0419)$, with the majority of children achieving vision of $6 / 60$ or better $(88.2 \%)$ and over one-third 6/12 or better.

Group B included 30 children with traumatic RD requiring vitrectomy. Silicone oil was injected in 20 $(66.7 \%)$, similar to the rate reported in another series of traumatic paediatric retinal detachments. ${ }^{21}$ The high rate of silicone oil use is due in part to the complexity of some traumatic RDs, but a second important factor is the expected compliance of children with postoperative posturing. We found no significant difference in the ages of children receiving oil or gas tamponade, but it is impossible to control for the complexity of the detachment in the analysis.

Full anatomic re-attachment of the retina was achieved with one procedure in $46.7 \%$ of eyes in group B. The visual results in this group were the most disappointing, with no change in the median VA from CF. About one third of eyes improved, one-third were stabilized, and one-third worsened, but $42.9 \%$ of children achieved a VA of $6 / 60$ or better at final follow-up. The anatomic and visual results in our series are better than those reported by Sarrazin $e a^{21}$ (33\% primary anatomic success, VA $\geq 6 / 60$ in $31 \%$ ).

Children in group $\mathrm{C}$ had attached retinas but underwent vitrectomy for a variety of other traumarelated indications, mainly opacities in the ocular media. No eye had RD at presentation and it is therefore not surprising that this group had the best anatomic and visual outcome.

Visual acuity was measured by the means most appropriate to the child's age and converted to the $6 \mathrm{~m}$ Snellen equivalent. Such measurements may be inaccurate in small children, particularly if the child is distressed. It is therefore possible that the preoperative acuities in particular may be underestimates of the true acuity at that time, and therefore exaggerate the apparent improvement at final follow-up.

The visual results in our patients with OGI are comparable to other published series. Sixty per cent of the children in this study achieved a final VA of $6 / 60$ or better, compared to between 56 and $74 \%$ reported in the literature. ${ }^{12,13}$ We were unable to find any literature reporting visual outcomes after CGI for comparison with our series.

There are many studies of the prognostic factors of OGI in adults. These are summarised in the report by the OTCG and include type of injury, VA, relative afferent pupillary defect, location of injury, wound size, vitreous haemorrhage, lenticular injury, RD, and endophthalmitis. ${ }^{14}$ Prognostic factors after OGI in children are less well defined. Features predictive of good visual outcome include age over 8 years, good initial VA, and small, anterior eyewall perforations. Poor prognostic factors include age less than 4 years, poor initial VA, large, posterior perforations, lenticular damage, and presence of RD. ${ }^{11-13}$

In this study, we defined poor outcome as CF or worse. We were unable to demonstrate any significant association between outcome and patient age, type of injury, presence of lens injury, vitreous haemorrhage, or endophthalmitis. The association between poor outcome and traumatic RD approached statistical significance $(P=0.0769)$. It is perhaps surprising that there was no significant association between visual outcome and, in particular, endophthalmitis or traumatic RD. The patients in this series were selected by their need for vitreoretinal surgery and therefore are likely to represent the more severe end of the paediatric trauma spectrum. In addition, the proportion of patients with ocular copathology (seven out of 61) is high. Both these factors may bias the results.

Factors significantly associated with poor outcome were preoperative VA CF or worse, presence of PVR, and traumatic RD requiring vitrectomy. Scleral lacerations 
extending posterior to the rectus muscle insertions carry a poor prognosis, and it is these injuries that are most likely to result in retinal detachment with vitreous haemorrhage or PVR and require vitrectomy. We had incomplete information regarding the posterior extent of the wound in the OGI group, but it is likely that the association between traumatic RD requiring vitrectomy and poor outcome is a surrogate marker for the severity of the ocular injury.

The presence of $\mathrm{RD}$ at final follow-up was also associated with poor outcome, but interestingly there was no association between outcome and number of surgical procedures required. This perhaps suggests that several attempts to re-attach the retina may be justified, but clearly the risk of multiple anaesthetics and the psychological effects of repeated procedures on the child and his or her family must also be taken into account.

PVR was a significant factor influencing the visual and anatomic outcome for the children in this study. About one third of eyes had retinal detachment complicated by PVR, and PVR was the cause of surgical failure in two thirds of cases. PVR is the only potentially modifiable risk factor for poor outcome identified by this study. Adjuvant treatment with 5-fluorouracil and heparin infusion has been shown to reduce the incidence of PVR in adult retinal detachment. ${ }^{22}$ Children with traumatic retinal detachment may also benefit from adjuvant treatment to reduce the incidence of this devastating complication, although further studies are required to confirm this.

\section{Conclusions}

It is not surprising that visual outcomes were best in those children whose injury did not result in traumatic RD. Scleral buckling for traumatic RD in children produced good anatomic and visual results and therefore should be considered in cases with clear media, anterior breaks, and no PVR. Vitrectomy for traumatic RD had the most disappointing outcomes, but this group self-selects the most severe injuries. PVR was a significant contributing factor to poor outcomes.

\section{References}

1 Katz J, Tielsch JM. Lifetime prevalence of ocular injuries from the Baltimore Eye Survey. Arch Ophthalmol 1993; 111: 1564-1568.

2 Klopfer J, Tielsch JM, Vitale S, See LC, Canner JK. Ocular trauma in the United States. Eye injuries resulting in hospitalisation, 1984 through 1987. Arch Ophthalmol 1992; 110: $838-842$.

3 Niiranen M, Raivio I. Eye injuries in children. $\mathrm{Br}$ J Ophthalmol 1981; 65: 436-438.
4 Zagelbaum BM, Tostanoski JR, Dawnielle JK, Hersh PS. Urban eye trauma. A one year prospective study. Ophthalmology 1993; 100: 851-856.

5 Schein OD, Hibberd PL, Shingleton BJ, Kunzweiler T, Frambach DA, Seddon JM et al. The spectrum and burden of ocular injury. Ophthalmology 1988; 95: 300-305.

6 Maltzman BA, Pruzon H, Mund ML. A survey of ocular trauma. Surv Ophthalmol 1976; 21: 285-290.

7 Mittra RA, Mieler WF. Controversies in the management of open globe injuries involving the posterior pole. Surv Ophthalmol 1999; 44: 215-225.

8 de Bustros S, Michels RG, Glaser BM. Evolving concepts in the management of posterior segment penetrating injuries. Retina 1990; 10: S72-S75.

9 Strahlman E, Elman M, Daub E, Baker S. Causes of pediatric eye injuries. A population-based study. Arch Ophthalmol 1990; 108: 603-606.

10 Kuhn F, Morris R, Witherspoon CD, Heimann K, Jeffers JB, Treister G. A standardized classification of ocular trauma. Ophthalmology 1996; 103: 240-243.

11 Baxter R, Hodgkins PR, Calder I, Morrell AJ, Vardy S, Elkington AR. Visual outcome of childhood anterior perforating eye injuries: prognostic indicators. Eye 1994; 8: 349-352.

12 Rudd JC, Jaeger EA, Freitag SK, Jeffers JB. Traumatically ruptured globes in children. J Pediatr Ophthalmol Strabismus 1994; 34: 307-311.

13 Farr AK, Hairston RJ, Humayun MU, Marsh MJ, Pieramici DJ, MacCumber MW et al. Open globe injuries in children: a retrospective analysis. J Pediatr Ophthalmol Strabismus 2001; 38: 72-77.

14 Pieramici DJ, Sternberg Jr P, Aaberg Sr TM, Bridges Jr WZ, Capone Jr A, Cardillo JA et al. A system for classifying mechanical injuries of the eye (globe). Am J Ophthalmol 1997; 123: 820-831.

15 Williams DF, Mieler WF, Williams GA. Posterior segment manifestations of ocular trauma. Retina 1990; 10: S35-S44.

16 Atmaca LS, Yilmaz M. Changes in the fundus caused by blunt ocular trauma. Ann Ophthalmol 1993; 25: 447-452.

17 Goffstein R, Burton TC. Differentiating traumatic from non-traumatic retinal detachments. Ophthalmology 1982; 89: 361-368.

18 Cleary PE, Ryan SJ. Method of production and natural history of experimental posterior penetrating eye injury in the rhesus monkey. Am J Ophthalmol 1979; 88: 212-220.

19 Häring G, Wiechens B. Long term results after scleral buckling surgery in uncomplicated juvenile retinal detachment without proliferative vitreoretinopathy. Retina 1998; 18: 501-505.

20 Sadeh AD, Dotan G, Bracha R, Lazar M, Loewenstein A. Characteristics and outcomes of paediatric rhegmatogenous retinal detachment treated by segmental scleral buckling plus an encircling element. Eye 2001; 15: 31-33.

21 Sarrazin L, Averbukh E, Halpert M, Hemo I, Rumelt S. Traumatic pediatric retinal detachment: a comparison between open and closed globe injuries. Am J Ophthalmol 2004; 137: 1042-1049.

22 Asaria RH, Kon CH, Bunce C, Charteris DG, Wong D, Khaw PT. Adjuvant 5-fluorouracil and heparin prevents proliferative vitreoretinopathy. Ophthalmology 2001; 108: 1179-1183. 